\title{
HÖLDER MEAN INEQUALITIES FOR THE GENERALIZED GRÖTZSCH RING AND HERSCH-PFLUGER DISTORTION FUNCTIONS
}

\author{
Song-Liang QiU, Ye-FAng QiU, MiaO-Kun WANG AND Yu-Ming Chu
}

Abstract. In this paper, we study the monotonicity properties of the generalized elliptic integrals and establish two Hölder mean inequalities for the generalized Grötzsch ring function $\mu_{a}(r)$ and the generalized Hersch-Pfluger distortion function $\varphi_{K}^{a}(r)$.

Mathematics subject classification (2010): 30C62, 33E05.

Keywords and phrases: Generalized elliptic integrals; generalized Grötzsch ring function; generalized Hersch-Pfluger distortion function; Hölder mean.

\section{REFERENCES}

[1] M. Abramowitz And I. A. Stegun, Handbook of Mathematical Functions with Formulas, Graphs, and Mathematical Tables, Dover Publications, New York, 1966.

[2] H. AlZER AND S. L. QIU, Monotonicity theorems and inequalities for the complete elliptic integrals, J. Comput. Appl. Math., 172, 2 (2004), 289-312.

[3] G. D. Anderson, S. L. Qiu, M. K. Vamanamurthy and M. Vuorinen, Generalized elliptic integrals and modular equations, Pacific J. Math., 192, 1 (2000), 1-37.

[4] G. D. Anderson, S. L. QIU And M. Vuorinen, Bounds for the Hersch-Pfluger and Belinskii distortion functions, Computational Methods and Function Theory 1997 (Nicosia), 9-22, Ser. Approx. Decompos., 11, World Sci. Publ., River Edge, NJ, 1999.

[5] G. D. Anderson, S. L. Qiu And M. Vuorinen, Precise estimates for differences of the Gaussian hypergeometric function, J. Math. Anal. Appl., 215, 1 (1997), 212-234.

[6] G. D. Anderson, M. K. Vamanamurthy and M. Vuorinen, Topics in special functions II, Confrom. Geom. Dyn., 11 (2007), 250-270.

[7] G. D. Anderson, M. K. Vamanamurthy and M. Vuorinen, Conformal Invariants, Inequalities, and Quasiconformal Maps, John Wiley \& Sons, New York, 1997.

[8] G. D. Anderson, M. K. VAmanamurthy AND M. Vuorinen, Inequalities for plane quasiconformal mappings, The Mathematical Legacy of Wilhelm Magnus: Groups, Geometry and Special Functions (Brooklyn, NY, 1992), 1-27, Contemp. Math., 169, Amer. Math. Soc., Providence, RI, 1994.

[9] G. D. Anderson, M. K. Vamanamurthy and M. Vuorinen, Functional inequalities for hypergeometric functions and complete elliptic integrals, SIAM J. Math. Anal., 23, 2 (1992), 512-524.

[10] G. D. Anderson, M. K. Vamanamurthy And M. Vuorinen, Functional inequalities for complete elliptic integrals and their ratios, SIAM J. Math. Anal., 21, 2 (1990), 536-549.

[11] G. D. Anderson, M. K. Vamanamurthy and M. Vuorinen, Distortion functions for plane quasiconformal mappings, Israel J. Math., 62, 1 (1988), 1-16.

[12] R. W. BARNARD, K. PEARCE AND K. C. Richards, A monotonicity property involving ${ }_{3} F_{2}$ and comparisons of the classical approximations of elliptical arc length, SIAM J. Math. Anal., 32, 2 (2000), 403-419.

[13] J. M. Borwein And P. B. Borwein, Pi and the AGM, John Wiley \& Sons, New York, 1987.

[14] P. S. Bullen, Handbook of Means and Their Inequalities, Kluwer Academatic Publishers Group, Dordrecht, 2003.

[15] S. Ponnus Amy AND M. VuORInen, Univalence and convexity properties for Gaussian hypergeometric functions, Rocky Mountain J. Math., 31, 1 (2001), 327-353. 
[16] S. L. Qiu, M. K. Vamanamurthy And M. Vuorinen, Some inequalities for the Hersch-Pfluger distortion function, J. Inequal. Appl., 4, 2 (1999), 115-139.

[17] S. L. QIU AND M. VuORINEN, Special functions in geometric function theory, Handbook of Complex Analysis: Geometric Function Theory, Vol. 2, 621-659, Elsevier Sci. B. V., Amsterdam, 2005.

[18] S. L. QIU AND M. VUORINEN, Landen inequalities for hypergeometric functions, Nagoya Math. J., 154 (1999), 31-56.

[19] S. L. QIU AND M. VUORINEN, Infinite products and normalized quotients of hypergeometric functions, SIAM J. Math. Anal., 30, 5 (1999), 1057-1075.

[20] M. Vuorinen, Conformal Geometry and Quasiregular Mappings, Springer-Verlag, Berlin, 1988. 